\title{
DEFORMATION OF CANTILEVER CURVED BEAM WITH VARIABLE CROSS SECTION
}

\author{
I. Ecsedi, A. BAKSA \\ University of Miskolc, Institute of Applied Mechanics, Hungary \\ istvan.ecsedi@uni-miskolc.hu attila.baksa@uni-miskolc.hu
}

[Received: October 31, 2020; Accepted: January 15, 2021]

Dedicated to Professor Barna Szabó on the occasion of his eighty-fifth birthday

\begin{abstract}
This paper deals with the determination of the displacements and stresses in a curved cantilever beam. The considered curved beam has circular centerline and the thickness of its cross section depends on the circumferential coordinate. The kinematics of Euler-Bernoulli beam theory are used. The curved elastic beam is fixed at one end and on the other end is subjected to concentrated moment and force; three different loading cases are considered. The paper gives analytical solutions for radial and circumferential displacements and cross-sectional rotation and circumferential stresses. The presented examples can be used as benchmark for the other types of solutions as given in this paper.
\end{abstract}

Mathematical Subject Classification: 74K10, 74B05

Keywords: curved beam, cantilever, variable cross section

\section{INTRODUCTION}

The analysis of curved beam has been a topic of interest to researchers for over a century, it is a standard topic in the most text books of mechanics [1 3$]$. This theme is still relevant at the present time because curved elements are important components in many modern engineering structures. In this paper an analytical solution is presented for cantilever curved beams with variable cross sections. One of the ends of a curved beam is fixed and the other end is subjected to radial and circumferential forces and a couple. Elasticity solutions are presented in [4] for curved beams with orthotropic functionally graded layers by means of Airy stress functions. The developed method is illustrated in curved cantilever beams with different types of loading conditions. Pydah and Sabele 5. present an analytical model for the flexure of bidirectional functionally graded circular beam. The formulation of the considered problem is based on the Euler-Bernoulli beam theory. The governing equations are solved for statically determinate circular cantilever beams under the action of tip loads. Paper $[6]$ deals with the determination of stress in circular curved beams with cross-sectional inhomogeneity. In Ecsedi and Lengyel 7 an analytical solution is presented for the determination of deformation of curved composite beams with uniform cross sections. The developed analytical solution is based on fundamental 
solutions which are filling to the given loading and boundary conditions. Closed form formulae are derived for the displacements, cross-sectional rotation normal and shear forces and bending moment. Paper 7] gives the expressions of circumferential and normal stresses and of shearing stress. Several studies give finite element numerical solutions to the in-plane deformation static problems of curved beams with uniform curvature such as 810 .

\section{Governing EQuations}

In the cylindrical coordinate system $\operatorname{Or} \varphi z$ the curved beam of variable cross section occupies the space domain (Figure 1)

$$
B=\left\{(r, \varphi, z)\left|R_{1} \leq r \leq R_{2},\right| z \mid \leq \frac{t(\varphi)}{2}, 0<\varphi<\alpha<2 \pi\right\},
$$

where $R_{i}(i=1,2)$ is the radius of inner and outer cylindrical boundary surface of body $B, t=t(\varphi)$ is the cross-sectional thickness in direction of axis $z$. The radius of the circular centerline of curved beam is

$$
r_{c}=\frac{1}{2}\left(R_{1}+R_{2}\right)
$$

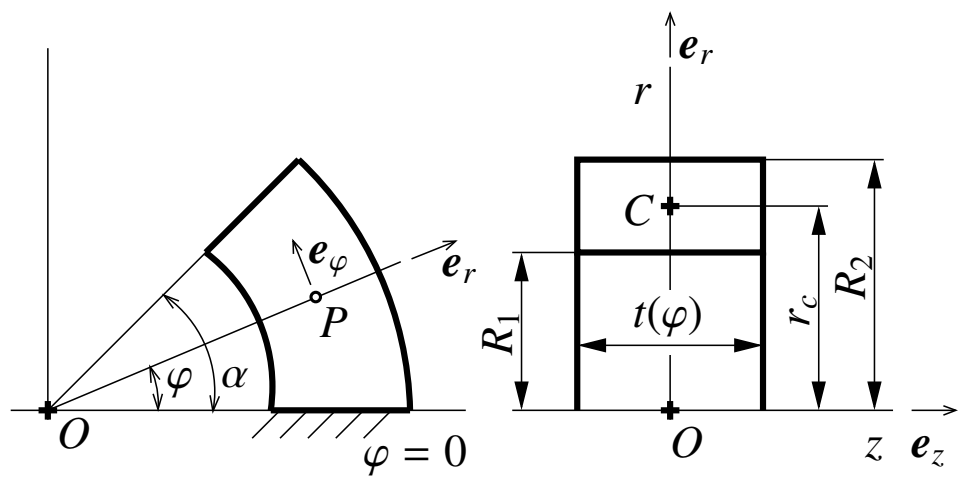

Figure 1. Cantilever curved beam and its cross section

The plane $z=0$ is the plane of symmetry of the curved beam and of the applied loads. Denote the unit vectors of cylindrical coordinate system $\operatorname{Or} \varphi z \boldsymbol{e}_{r}, \boldsymbol{e}_{\varphi}$ and $\boldsymbol{e}_{z}$. We start from the next displacement field to describe the in-plane deformation of cantilever curved beam 11

$$
\begin{gathered}
\boldsymbol{u}=u \boldsymbol{e}_{r}+v \boldsymbol{e}_{\varphi}+w \boldsymbol{e}_{z}, \\
u=U(\varphi), \quad v=r \phi(\varphi)+V(\varphi), \quad V(\varphi)=\frac{\mathrm{d} U}{\mathrm{~d} \varphi}, \quad w=0 .
\end{gathered}
$$


Application of the strain-displacement relationships of the linearized theory of elasticity gives 12,13

$$
\begin{gathered}
\varepsilon_{r}=\frac{\partial u}{\partial r}=0, \quad \varepsilon_{z}=\frac{\partial w}{\partial z}=0, \quad \gamma_{r \varphi}=\frac{1}{r}\left(\frac{\partial u}{\partial \varphi}-v\right)=0, \quad \gamma_{\varphi z}=\frac{\partial v}{\partial z}+\frac{1}{r} \frac{\partial w}{\partial \varphi}=0, \\
\gamma_{r z}=\frac{\partial u}{\partial z}+\frac{\partial w}{\partial r}=0, \quad \varepsilon_{\varphi}=\frac{W(\varphi)}{r}+\frac{\mathrm{d} \phi}{\mathrm{d} \varphi}, \quad W(\varphi)=\frac{\mathrm{d}^{2} U}{\mathrm{~d} \varphi^{2}}+U .
\end{gathered}
$$

The strain field given by equations (2.5), 2.6) satisfy the requirements of the BernoulliEuler beam theory, only the normal strain $\varepsilon_{\varphi}$ is different from zero and all the other strains vanish. Based on paper [11] we define the stress resultant forces $N=N(\varphi)$, $S=S(\varphi)$ and stress couple resultant $M=M(\varphi)$ as

$$
N(\varphi)=\int_{A(\varphi)} \sigma_{\varphi} \mathrm{d} A, \quad S(\varphi)=\int_{A(\varphi)} \tau_{r \varphi} \mathrm{d} A, \quad M(\varphi)=\int_{A(\varphi)} r \sigma_{\varphi} \mathrm{d}(A),
$$

where $\tau_{r \varphi}=\tau_{r \varphi}(r, \varphi)$ denotes the shearing stress. Here, we note, the shear force $S=S(\varphi)$ will be computed by the use of equilibrium equation which is the usual in the case of Euler-Bernoulli beam theory. Figure 2 illustrates the stress and stress couple resultants in an arbitrary cross section of curved beam. Application of Hooke's law yields the formula of normal stresses $\sigma_{\varphi}[13$

$$
\sigma_{\varphi}=E\left(\frac{W(\varphi)}{r}+\frac{\mathrm{d} \phi}{\mathrm{d} \varphi}\right)
$$

where $E$ is the modulus of elasticity. From equations $(2.7)$ it follows that

$$
\begin{aligned}
& N(\varphi)=E A(\varphi)\left(\frac{W(\varphi)}{R}+\frac{\mathrm{d} \phi}{\mathrm{d} \varphi}\right), \\
& M(\varphi)=E A(\varphi)\left(W(\varphi)+r_{c} \frac{\mathrm{d} \phi}{\mathrm{d} \varphi}\right) .
\end{aligned}
$$

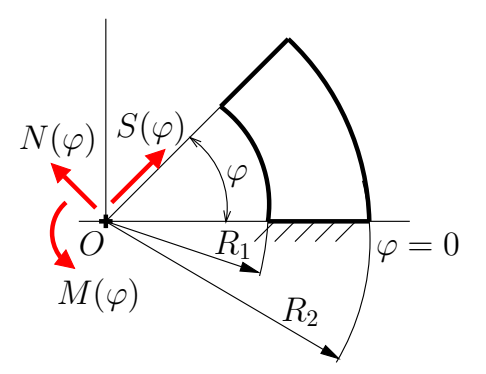

Figure 2. Illustration of $N(\varphi), S(\varphi)$ and $M(\varphi)$

In equations 2.9$), 2.10) A=t(\varphi)\left(R_{2}-R_{1}\right)$ is the area of the cross section, $r_{c}=\frac{1}{2}\left(R_{1}+R_{2}\right)$ is the radial coordinate of the center of cross section (Figure 1 and

$$
R=\frac{R_{2}-R_{1}}{\ln \frac{R_{2}}{R_{1}}}
$$


The equilibrium equations in terms of $N, S$ and $M$ for curved cantilever beam which is loaded its end cross sections are 7,11

$$
\frac{\mathrm{d} N}{\mathrm{~d} s}+S=0, \quad \frac{\mathrm{d} S}{\mathrm{~d} \varphi}-N=0, \quad \frac{\mathrm{d} M}{\mathrm{~d} \varphi}=0 .
$$

We remark that the considered cantilever curved beams satisfy the boundary conditions

$$
U(0)=0, \quad V(0)=0, \quad \phi(0)=0 .
$$

\section{Cantilever Curved BeAm LOAded By Bending MOMEnT}

Figure 3 shows the cantilever curved beam, which is loaded at its end cross section $\varphi=\alpha$ by a bending moment $M_{1}$. From equilibrium equations 2.12 we have

$$
N(\varphi)=S(\varphi)=0, \quad M(\varphi)=M_{1}=\text { constant }, \quad \varphi_{1} \leq \varphi \leq \varphi_{2} .
$$

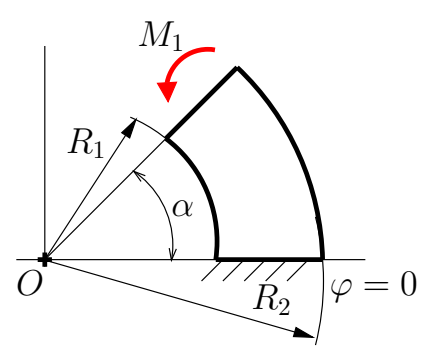

Figure 3. Cantilever curved beam with bending moment

Combination of equations 2.9 and 2.10 with equation 3.1 gives

$$
\begin{gathered}
W_{1}(\varphi)+R \frac{\mathrm{d} \phi_{1}}{\mathrm{~d} \varphi}=0, \\
W_{1}(\varphi)+r_{c} \frac{\mathrm{d} \phi_{1}}{\mathrm{~d} \varphi}=\frac{M_{1}}{E A(\varphi)} .
\end{gathered}
$$

The solution of the system of equations $3.2,3.3$ for $W_{1}(\varphi)$ and $\frac{\mathrm{d} \phi_{1}}{\mathrm{~d} \varphi}$ is as follows:

$$
W_{1}(\varphi)=-\frac{M_{1}}{e E A(\varphi)}, \quad \frac{\mathrm{d} \phi_{1}}{\mathrm{~d} \varphi}=\frac{M_{1}}{e E A(\varphi)}, \quad e=r_{c}-R .
$$

In the present problem

$$
W_{1}(\varphi)=\frac{\mathrm{d}^{2} U_{1}}{\mathrm{~d} \varphi^{2}}+U_{1}
$$

Here, we will use the result of the theory of ordinary differential equations 14,15 : 
Theorem 1: Let $h=h(x)$ be a continuous bounded function defined for $x \in$ $[0, a>0]$. In this case the solution of the initial value problem

$$
\frac{\mathrm{d}^{2} y}{\mathrm{~d} x^{2}}+y=h(x), \quad 0 \leq x \leq a, \quad y(0)=0,\left.\quad \frac{\mathrm{d} y}{\mathrm{~d} x}\right|_{x=0}=0,
$$

can be represented as

$$
y(x)=\int_{0}^{x} h(\lambda) \sin (x-\lambda) \mathrm{d} \lambda
$$

and we have

$$
\frac{\mathrm{d} y}{\mathrm{~d} x}=\int_{0}^{x} h(\lambda) \cos (x-\lambda) \mathrm{d} \lambda .
$$

The application of Theorem 1 gives the next formulae for $U_{1}=U_{1}(\varphi)$ and $V_{1}=$ $V_{1}(\varphi)$

$$
\begin{gathered}
U_{1}(\varphi)=-\frac{M_{1} R}{e\left(R_{2}-R_{1}\right) E} \int_{0}^{\varphi} \frac{\sin (\varphi-\vartheta)}{t(\vartheta)} \mathrm{d} \vartheta, \\
V_{1}=\frac{\mathrm{d} U_{1}}{\mathrm{~d} \varphi}=-\frac{M R}{e\left(R_{2}-R_{1}\right) E} \int_{0}^{\varphi} \frac{\cos (\varphi-\vartheta)}{t(\vartheta)} \mathrm{d} \vartheta .
\end{gathered}
$$

A direct integration of equation (3.4) yields the result

$$
\phi_{1}(\varphi)=\frac{M_{1}}{e\left(R_{2}-R_{1}\right) E} \int_{0}^{\varphi} \frac{\mathrm{d} \vartheta}{t(\vartheta)} .
$$

By the use of formula 2.8 we get the expression of circumferential normal stress

$$
\sigma_{\varphi}=\frac{M_{1}}{e\left(R_{2}-R_{1}\right)} \frac{r-R}{r t(\varphi)}
$$

The circumferential normal stress $\sigma_{\varphi}$ is zero in all cross sections at the radial coordinate $r=R$.

\section{Cantilever Curved beam loaded by Radial force}

The cantilever curved beam which is loaded at its end cross section $\varphi=\alpha$ by a radial force $F_{2}$ is shown in Figure 4. From equilibrium equations in this case we have

$$
N(\varphi)=-F \sin (\varphi-\alpha), \quad S(\varphi)=F \cos (\varphi-\alpha), \quad M(\varphi)=0, \quad 0 \leq \varphi \leq \alpha .
$$

In the present problem the application of equations 2.9 and 2.10 gives

$$
\begin{gathered}
W_{2}(\varphi)+R \frac{\mathrm{d} \phi_{2}}{\mathrm{~d} \varphi}=-\frac{F_{2} R}{E A(\varphi)} \sin (\varphi-\alpha), \\
W_{2}(\varphi)+r_{c} \frac{\mathrm{d} \phi_{2}}{\mathrm{~d} \varphi}=0 .
\end{gathered}
$$




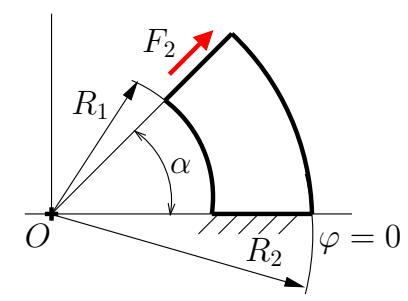

Figure 4. Cantilever curved beam subjected by radial force

Solution of the system of equations for $W_{2}=W_{2}(\varphi)$ and $\frac{\mathrm{d} \phi_{2}}{\mathrm{~d} \varphi}$ is as follows

$$
W_{2}(\varphi)=-\frac{F_{2} R r_{2}}{E A(\varphi) e} \sin (\varphi-\alpha), \quad \frac{\mathrm{d} \phi_{2}}{\mathrm{~d} \varphi}=\frac{F_{2} R}{E A(\varphi) e} \sin (\varphi-\alpha) .
$$

Substitution of the expression $W_{2}=W_{2}(\varphi)$ into equation 3.6 gives the formula of radial displacement function $U_{2}=U_{2}(\varphi)$

$$
U_{2}(\varphi)=-\frac{F_{2} R r_{c}}{e\left(R_{2}-R_{1}\right) E} \int_{0}^{\varphi} \sin (\varphi-\alpha) \frac{\sin (\vartheta-\alpha)}{t(\vartheta)} \mathrm{d} \vartheta .
$$

A simple computation based on equation 4.5 yields the result

$$
V_{2}(\varphi)=\frac{\mathrm{d} U_{2}}{\mathrm{~d} \varphi}=-\frac{F_{2} R r_{c}}{e\left(R_{2}-R_{1}\right) E} \int_{0}^{\varphi} \cos (\varphi-\alpha) \frac{\sin (\vartheta-\alpha)}{t(\vartheta)} \mathrm{d} \vartheta
$$

From equation (4.4) it follows that

$$
\phi_{2}(\varphi)=\frac{F_{2} R}{E\left(R_{2}-R_{1}\right) e} \int_{0}^{\varphi} \frac{\sin (\vartheta-\alpha)}{t(\vartheta)} \mathrm{d} \vartheta .
$$

In the present case we obtain for the circumferential normal stress the formula

$$
\sigma_{\varphi}=\frac{F_{2} R}{\left(R_{2}-R_{1}\right) e} \frac{r-r_{c}}{r} \frac{\sin (\varphi-\alpha)}{t(\varphi)} .
$$

The circumferential normal stress $\sigma_{\varphi}$ is zero in all cross sections at the radial coordinate $r=r_{c}$.

\section{Cantilever Curved Beam loaded by normal force}

The curved beam which is fixed at one end and loaded by normal force $F_{3}$ at the other end is shown in Figure 5 . In this case the solutions of equilibrium equations are as follows:

$$
N(\varphi)=F_{3} \cos (\varphi-\alpha), \quad S(\varphi)=-F_{3} \sin (\varphi-\alpha), \quad M(\varphi)=0, \quad 0 \leq \varphi \leq \alpha .
$$




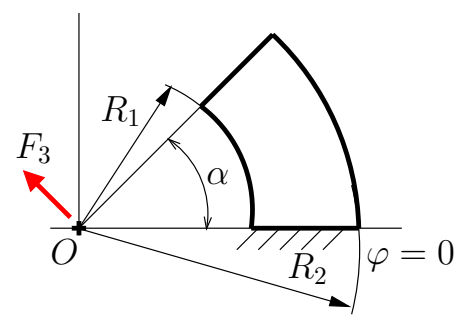

Figure 5. Cantilever curved beam loaded by normal force

In the present problem equations 2.9 and 2.10 lead to the system of equations for $W_{3}=W_{3}(\varphi)$ and $\frac{\mathrm{d} \phi_{3}}{\mathrm{~d} \varphi}$

$$
\begin{gathered}
W_{3}(\varphi)+R \frac{\mathrm{d} \phi_{3}}{\mathrm{~d} \varphi}=\frac{F_{3} R}{E A} \cos (\varphi-\alpha), \\
W_{3}(\varphi)+r_{c} \frac{\mathrm{d} \phi_{3}}{\mathrm{~d} \varphi}=0 .
\end{gathered}
$$

A simple computation gives

$$
W_{3}(\varphi)=\frac{F_{3} R r_{c}}{E e A(\varphi)} \cos (\varphi-\alpha), \quad \frac{\mathrm{d} \phi_{3}}{\mathrm{~d} \varphi}=-\frac{F_{3} R}{\operatorname{EeA}(\varphi)} \cos (\varphi-\alpha) .
$$

Application of Theorem 1 yields the expression of radial displacement $U_{3}=U_{3}(\varphi)$

$$
U_{3}(\varphi)=\frac{F_{3} R r_{c}}{E e\left(R_{2}-R_{1}\right)} \int_{0}^{\varphi} \frac{\cos \vartheta-\alpha}{t(\vartheta)} \sin (\varphi-\vartheta) \mathrm{d} \vartheta .
$$

The circumferential displacement $V_{3}=V_{3}(\varphi)$ is

$$
V_{3}(\varphi)=\frac{\mathrm{d} U_{3}}{\mathrm{~d} \varphi}=\frac{F_{3} R}{E e\left(R_{2}-R_{1}\right)} \int_{0}^{\varphi} \frac{\cos (\vartheta-\alpha)}{t(\varphi)} \cos (\varphi-\vartheta) \mathrm{d} \vartheta .
$$

Integration of equation 5.4 ) gives the cross-sectional rotation function $\phi_{3}=\phi_{3}(\varphi)$

$$
\phi_{3}(\varphi)=-\frac{F_{3} R}{E e\left(R_{2}-R_{1}\right)} \int_{0}^{\varphi} \frac{\cos (\vartheta-\alpha)}{t(\vartheta)} \mathrm{d} \vartheta .
$$

From equation 2.8 we obtain the formula of the circumferential normal stress $\sigma_{\varphi}$ as

$$
\sigma_{\varphi}=\frac{F_{3} R}{e\left(R_{2}-R_{1}\right)} \frac{r_{c}-r}{r} \frac{\cos (\varphi-\alpha)}{t(\varphi)} .
$$

Here, we note that the circumferential normal stress $\sigma_{\varphi}$ is zero in all cross sections at the radial coordinate $r=r_{c}$. 


\section{Betti's THEOREM FOR CANTILEVER CURVED BEAM}

Let us consider two different equilibrium states of cantilever curved beam with end loads. The applied loads for the first equilibrium state are $M_{1}^{\prime}, F_{2}^{\prime}$ and $F_{3}^{\prime}$ which cause the displacements $\widetilde{U}(\varphi), \widetilde{V}(\varphi)$ and $\widetilde{\phi}(\varphi)$. The applied loads for the second equilibrium state are $M_{1}^{\prime \prime}, F_{2}^{\prime \prime}$ and $F_{3}^{\prime \prime}$ and the corresponding displacements to the end cross sectional loads $M_{1}^{\prime \prime}, F_{2}^{\prime \prime}$ and $F_{3}^{\prime \prime}$ are ${ }^{*}(\varphi), \stackrel{*}{V}(\varphi)$ and $\stackrel{*}{\phi}(\varphi)$. A simple computation shows that the work done by system of forces on the displacement field $\stackrel{*}{U}(\varphi), \stackrel{*}{V}(\varphi)$ and $\stackrel{*}{\phi}(\varphi)$ is as follows

$$
w_{12}=\left.\int_{A} \sigma_{\varphi}^{\prime}(r \stackrel{*}{\phi}+\stackrel{*}{V})\right|_{\varphi=\alpha} \mathrm{d} A+\left.\int_{A} \tau_{r \varphi}^{\prime} \stackrel{*}{U}\right|_{\varphi=\alpha} \mathrm{d} A=M_{1}^{\prime} \phi(\alpha)+F_{3}^{\prime} V(\alpha)+F_{2}^{\prime} \stackrel{*}{U}(\alpha) .
$$

It is evident the work done by system of forces $M_{1}^{\prime \prime}, F_{2}^{\prime \prime}$ and $F_{3}^{\prime \prime}$ on the displacement field $\widetilde{U}(\varphi), \widetilde{V}(\varphi)$ and $\widetilde{\phi}(\varphi)$ is obtained from the equation

$$
w_{21}=M_{1}^{\prime \prime} \widetilde{\phi}(\alpha)+F_{3}^{\prime \prime} \widetilde{V}(\alpha)+F_{2}^{\prime \prime} \widetilde{U}(\alpha) .
$$

According to Betti's theorem [12, 13 we have

$$
w_{12}=w_{21} \text {. }
$$

We will use Betti's theorem for the following three equilibrium states

$$
\begin{gathered}
M_{1}^{\prime}=M_{1}, \quad F_{2}^{\prime}=F_{3}^{\prime}=0, \quad \widetilde{U}(\varphi)=U_{1}(\varphi), \quad \tilde{V}(\varphi)=V_{1}(\varphi), \quad \widetilde{\phi}(\varphi)=\phi_{1}(\varphi) ; \\
M_{1}^{\prime \prime}=0, \quad F_{2}^{\prime \prime}=F_{2}, \quad F_{3}^{\prime \prime}=0, \quad \stackrel{*}{U}(\varphi)=U_{2}(\varphi), \quad \stackrel{*}{V}(\varphi)=V_{2}(\varphi), \quad \stackrel{*}{\phi}=\phi_{2}(\varphi) .
\end{gathered}
$$

We remark that the third equilibrium state is defined as

$$
M_{1}^{\prime \prime \prime}=0, \quad F_{2}^{\prime \prime \prime}=0, \quad F_{3}^{\prime \prime \prime}=F_{2}, \quad \hat{U}(\varphi)=U_{3}(\varphi), \quad \hat{V}(\varphi)=V_{3}(\varphi), \quad \hat{\phi}=\phi_{3}(\varphi) .
$$

For these equilibrium states the following equations must be valid according to Betti's theorem:

$$
w_{12}=w_{21}, \quad w_{13}=w_{31}, \quad w_{23}=w_{32} .
$$

In the present problems we have

$$
\begin{aligned}
& w_{12}=M_{1} \phi_{2}(\alpha)=\frac{M_{1} F_{2} R}{E\left(R_{2}-R_{1}\right) e} \int_{0}^{\alpha} \frac{\sin (\vartheta-\alpha)}{t(\vartheta)} \mathrm{d} \vartheta \\
& w_{21}=F_{2} U_{1}(\alpha)=-\frac{F_{2} M_{1} R}{E\left(R_{2}-R_{1}\right) e} \int_{0}^{\alpha} \frac{\sin (\alpha-\vartheta)}{t(\vartheta)} \mathrm{d} \vartheta \\
& w_{13}=M_{1} \phi_{3}(\alpha)=-\frac{M_{1} F_{3} R}{E\left(R_{2}-R_{1}\right) e} \int_{0}^{\alpha} \frac{\cos (\vartheta-\alpha)}{t(\vartheta)} \mathrm{d} \vartheta \\
& w_{31}=F_{3} V_{1}(\alpha)=-\frac{M_{1} F_{3} R}{E\left(R_{2}-R_{1}\right) e} \int_{0}^{\alpha} \frac{\cos (\alpha-\vartheta)}{t(\vartheta)} \mathrm{d} \vartheta
\end{aligned}
$$




$$
\begin{aligned}
& w_{23}=F_{2} U_{3}(\alpha)= \\
& =\frac{F_{2} F_{3} R r_{c}}{E\left(R_{2}-R_{1}\right) e} \int_{0}^{\alpha} \frac{\cos (\vartheta-\alpha)}{t(\vartheta)} \sin (\alpha-\vartheta) \mathrm{d} \vartheta \\
& w_{32}=F_{3} V_{2}(\alpha)= \\
& =-\frac{F_{2} F_{3} R r_{c}}{E\left(R_{2}-R_{1}\right) e} \int_{0}^{\alpha} \frac{\cos (\alpha-\vartheta)}{t(\vartheta)} \sin (\vartheta-\alpha) \mathrm{d} \vartheta
\end{aligned}
$$

The validity of equations $6.4{ }_{1,2,3}$ follows from equations 6.56 .10 .

\section{NUMERICAL EXAMPLES}

The following data are used in the numerical example: $E=2 \times 10^{5} \mathrm{MPa}$, $R_{1}=0.015 \mathrm{~m}, R_{2}=0.025 \mathrm{~m}, t_{0}=0.015 \mathrm{~m}, \alpha=\frac{\pi}{3}, t(\varphi)=t_{0} \cos \varphi$.

7.1. Example 1. (see Figure 3) $M_{1}=50 \mathrm{Nm}, F_{2}=F_{3}=0$ The graphs of the radial displacement $U_{1}=U_{1}(\varphi)$ and circumferential displacement $V_{1}=V_{1}(\varphi)$ are shown in Figure 6] and the cross sectional rotation $\phi_{1}=\phi_{1}(\varphi)$ is presented in Figure 7. The plots of $\sigma_{\varphi}(r, \varphi)$ for four different values of polar angle $\varphi\left(\varphi=0, \varphi=\frac{\pi}{6}, \varphi=\frac{\pi}{4}, \varphi=\frac{\pi}{3}\right)$ are shown in Figure 8 .

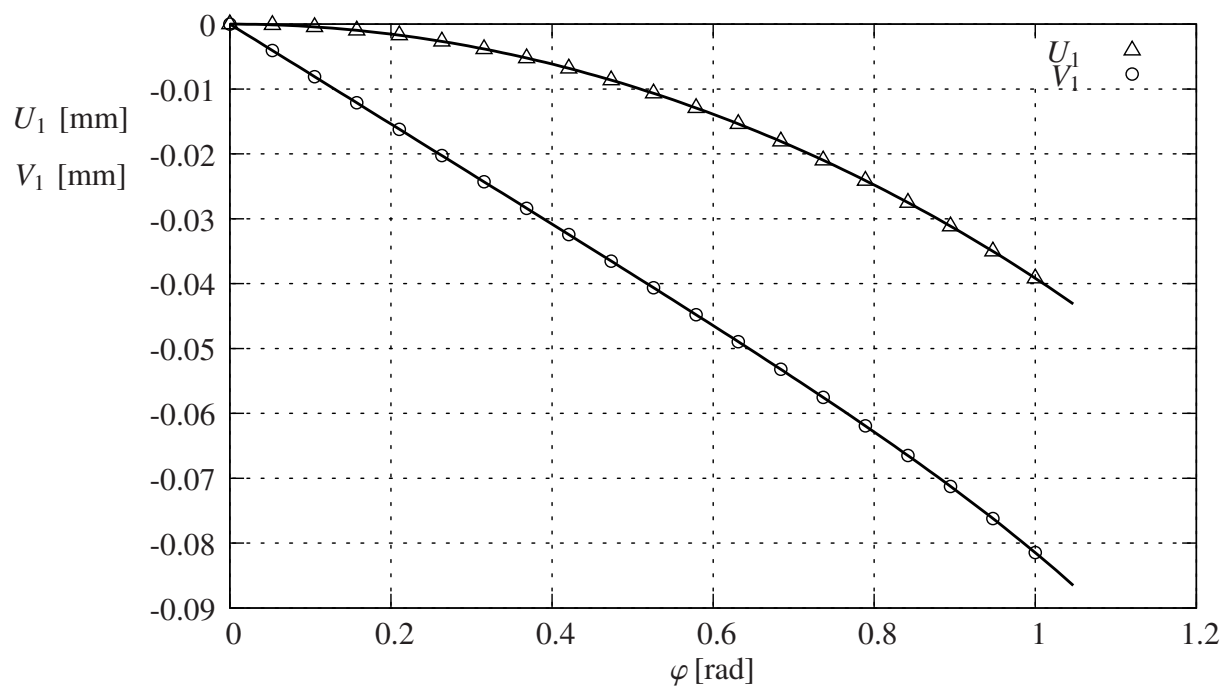

Figure 6. Plots of $U_{1}$ and $V_{1}$ 


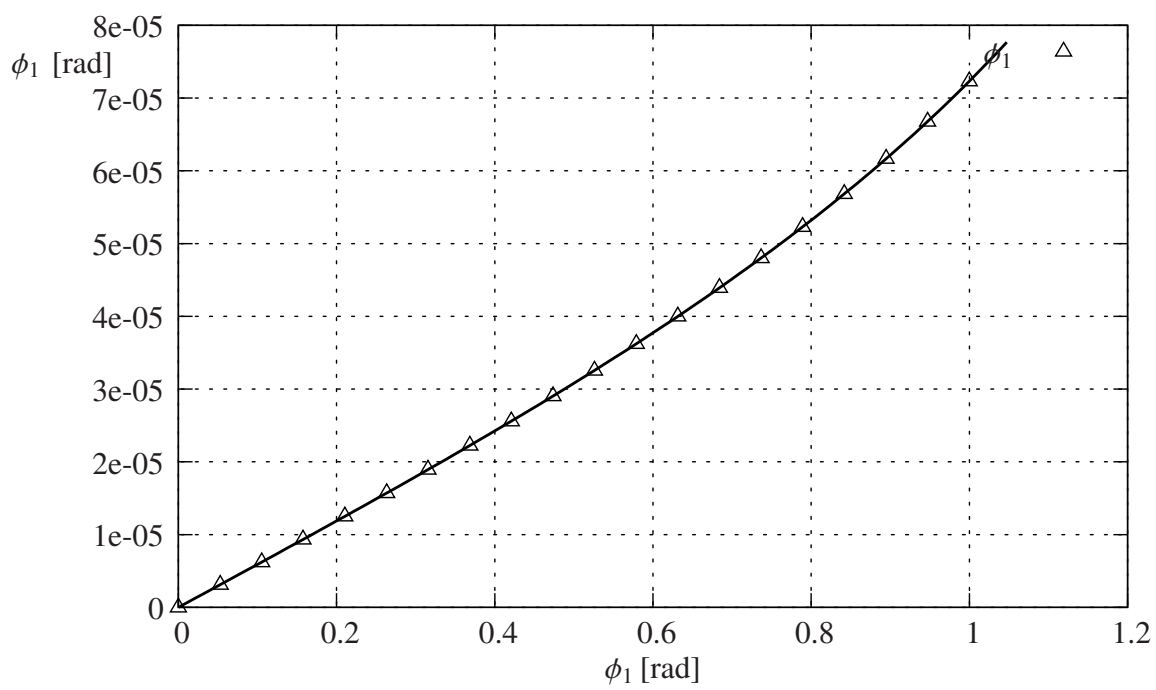

Figure 7. Plot of $\phi_{1}$

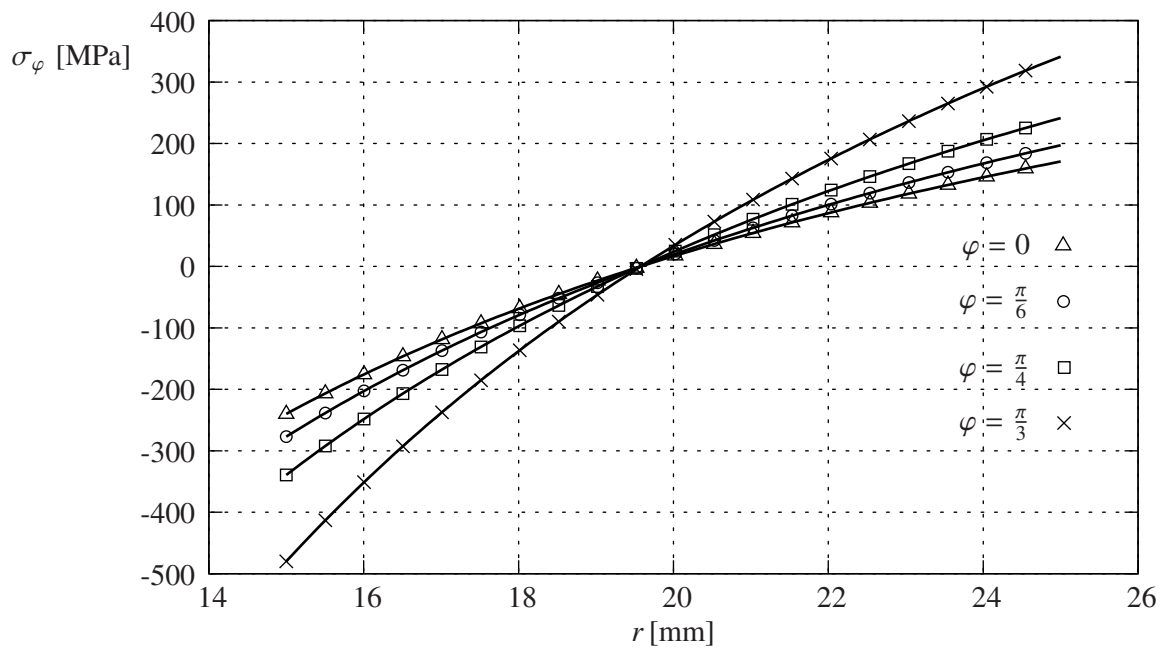

Figure 8. The plots of $\sigma_{\varphi}$ (Example 1)

7.2. Example 2. (see Figure 4) $F_{2}=1400 \mathrm{~N}, M_{1}=0, F_{3}=0$. The graphs of radial displacement $U_{2}=U_{2}(\varphi)$ and circumferential displacement $V_{2}=V_{2}(\varphi)$ are given in Figure 9. The plots of cross-sectional rotation $\phi_{2}=\phi_{2}(\varphi)$ is shown in Figure 10. The plots of the circumferential stress $\sigma_{\varphi}(r, \varphi)$ for four different values of polar angle $\varphi\left(\varphi=0, \varphi=\frac{\pi}{6}, \varphi=\frac{\pi}{4}, \varphi=\frac{\pi}{3}\right)$ are shown in Figure 11 


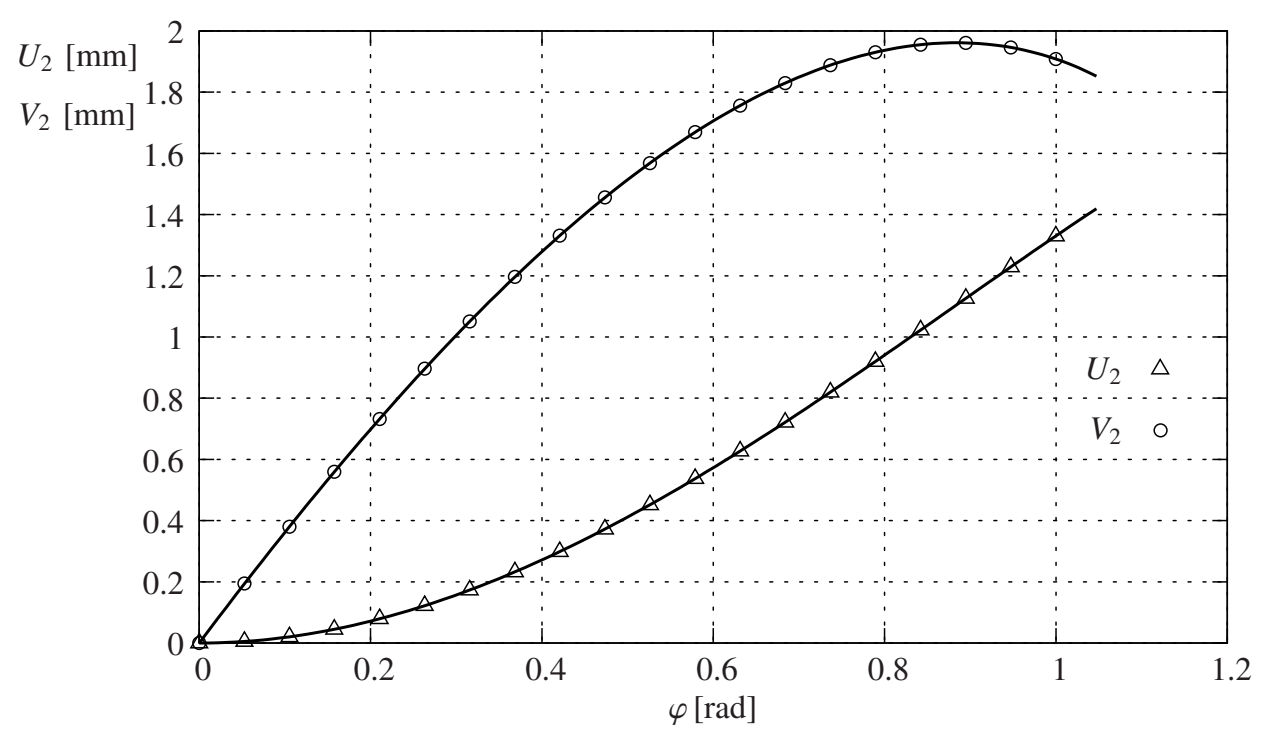

Figure 9. Plots of $U_{2}$ and $V_{2}$

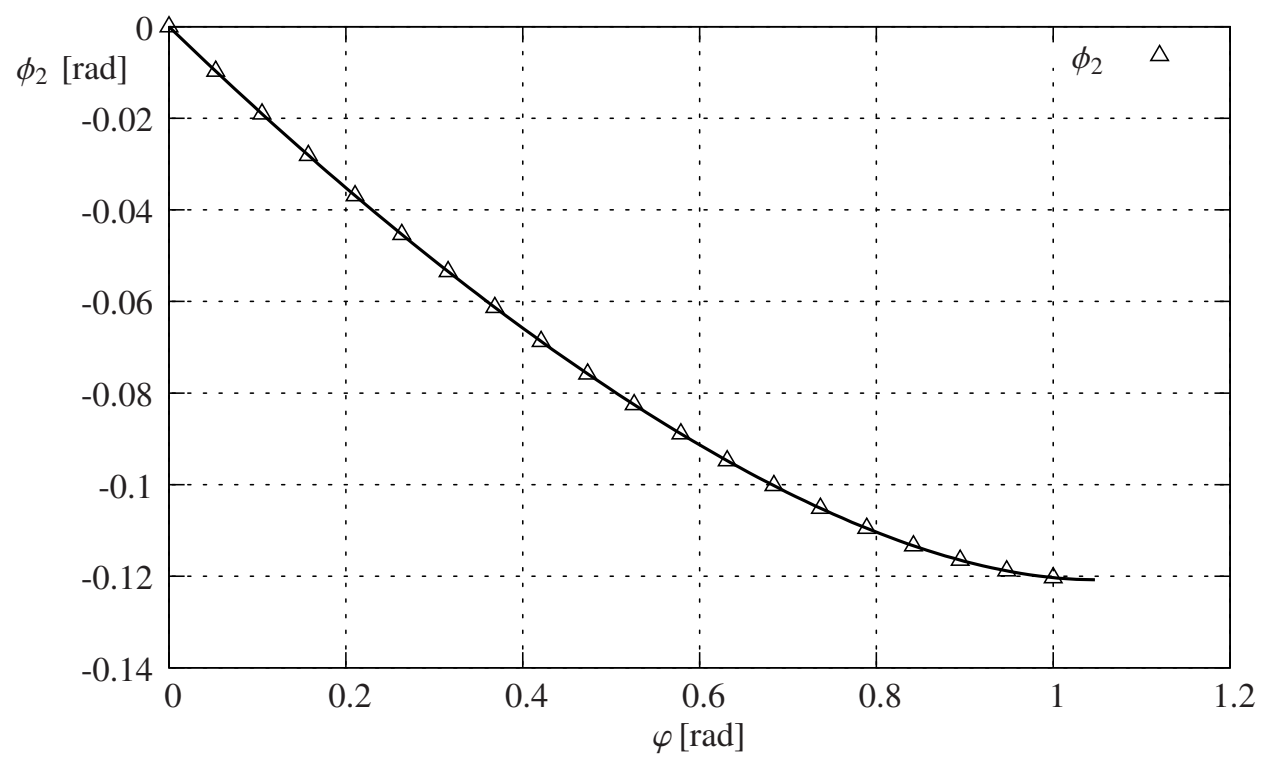

Figure 10. Plot of $\phi_{2}$ 


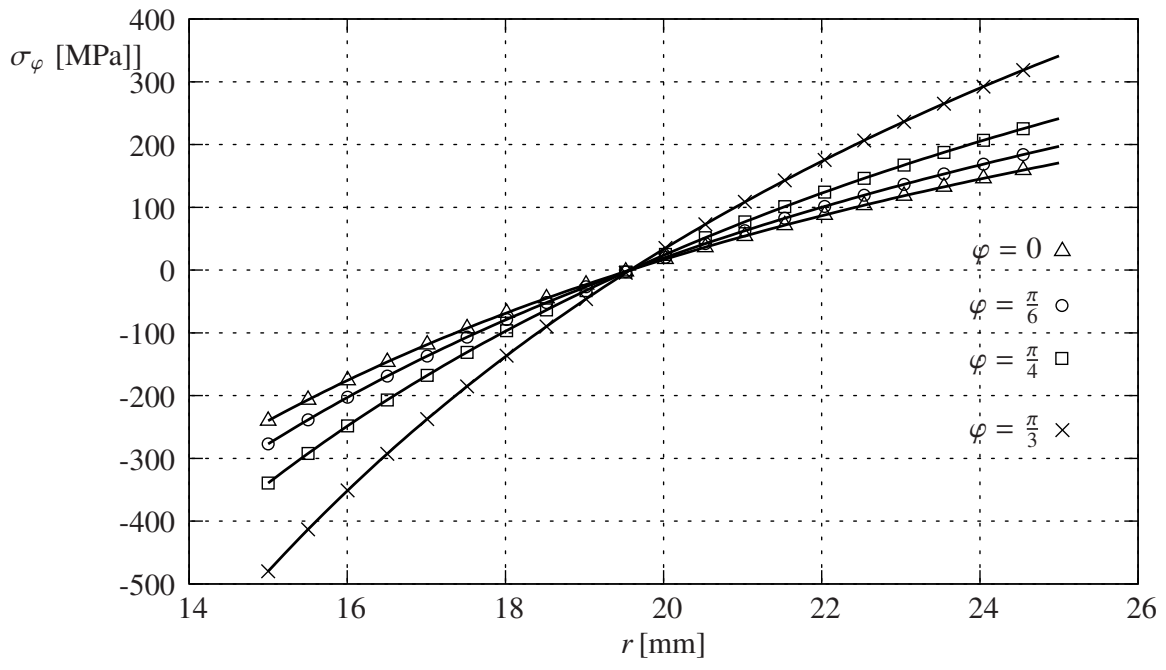

Figure 11. Plots of $\sigma_{\varphi}$

7.3. Example 3. (see Figure 5) $F_{3}=1000 \mathrm{~N}, M_{1}=0, F_{2}=0$. In this case the plots of radial displacement $U_{3}=U_{3}(\varphi)$, and circumferential displacement $V_{3}=V_{3}(\varphi)$ are given in Figure 12. The graph of $\phi_{3}=\phi_{3}(\varphi)$ is presented in Figure 13. The plots of circumferential stress $\sigma_{\varphi}(r, \varphi)$ for four different values of the polar angle $\varphi$ $\left(\varphi=0, \varphi=\frac{\pi}{6}, \varphi=\frac{\pi}{4}, \varphi=\frac{\pi}{3}\right)$ are shown in Figure 14

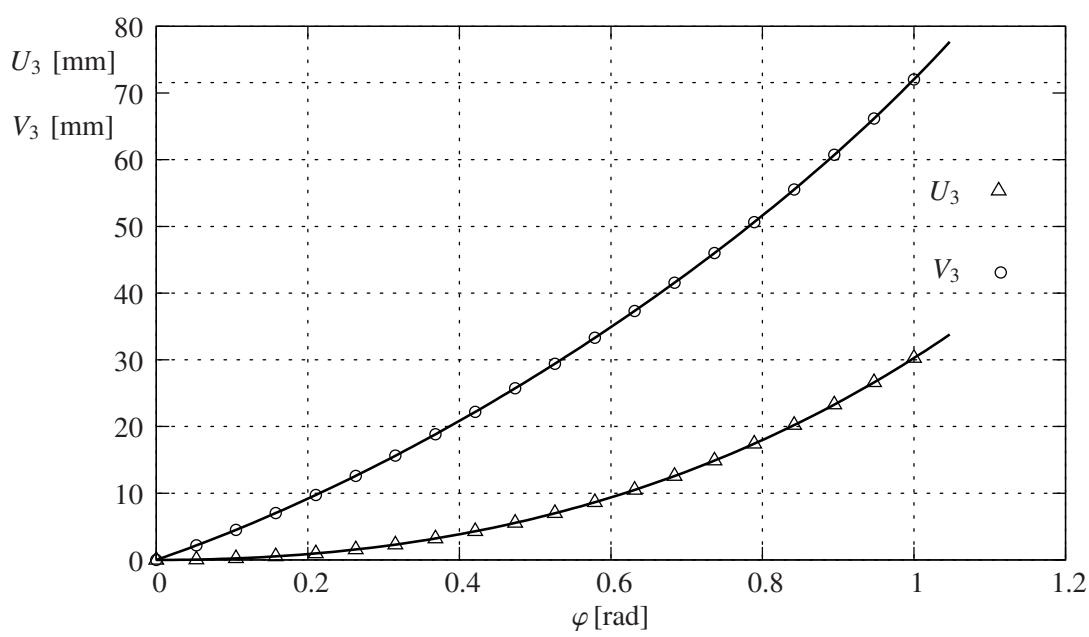

Figure 12. Plots of $U_{3}$ and $V_{3}$. 


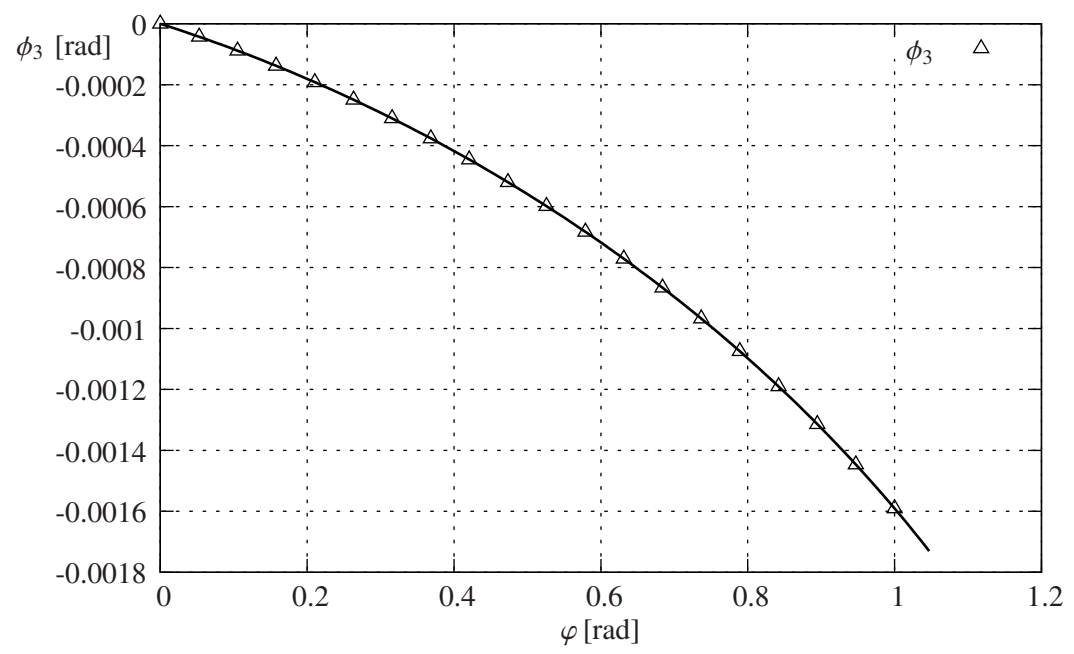

Figure 13. Plot of $\phi_{3}$

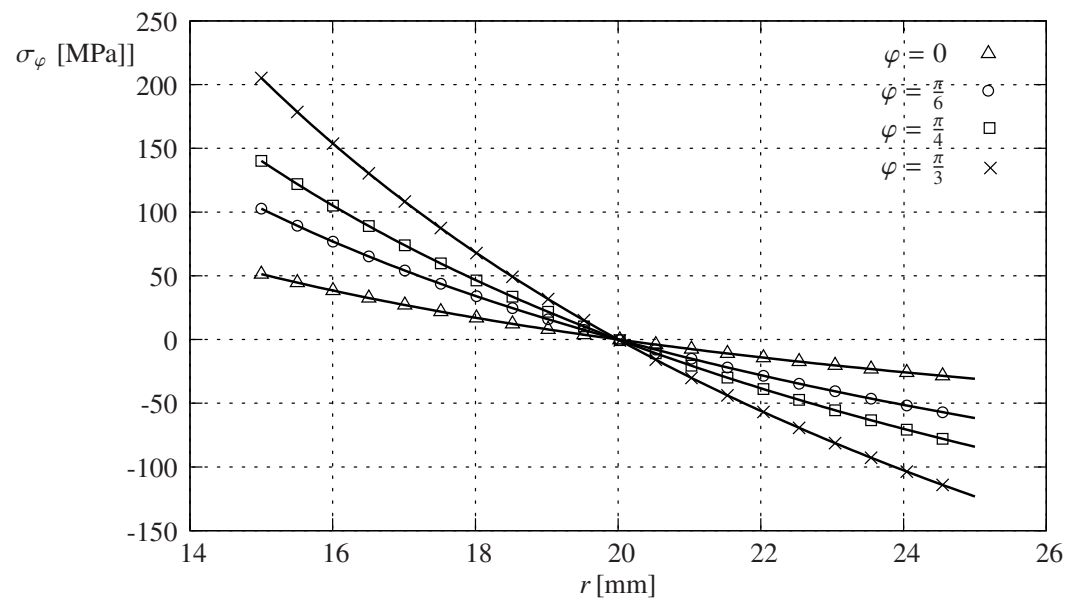

Figure 14. Plots of $\sigma_{\varphi}$

\section{Conclusions}

In this paper an elastic cantilever curved beam of variable cross section is studied. At one end of the beam its cross section is fixed and at the other end it is loaded by concentrated forces and couples. Three different loading cases are considered. The paper presents an analytical solution to obtain the radial and circumferential displacements, cross sectional rotation and circumferential normal stress. Formulation of the considered equilibrium problems is based on the Euler-Bernoulli beam theory. 
The validity of the obtained result is supported by Betti's theorem. Three numerical examples illustrate the application of the developed analytical solutions.

Acknowledgement. The present research was partially supported by the Hungarian Academy of Sciences, by grant NKFIH 115701.

\section{REFERENCES}

1. A. P. Boresi and R. J. Schmidt. Advanced Mechanics of Materials. Wiley and Sons, Inc., New York, 2003. DOI: 10.1111/j.1475-1305.1993.tb00852.x.

2. R. Solecki and R. J. Conant. Advanced Mechanics of Materials. Oxford University Press, Oxford, 2003.

3. R. D. Cook and W. C. Young. Advanced Mechanics of Materials. Macmillan, New York, 1985.

4. M. Wang and Y. Liu. "Elasticity solutions for orthotropic functinally graded curved beams." European Journal of Mechanics-A/Solids, 37, (2013), pp. 8-13. DOI: $10.1016 / \mathrm{j}$.euromechsol.2012.04.005.

5. A. Pydah and A. Sabela. "Static analysis of bidirectional functionally graded beams." Composite Structures, 160, (2017), pp. 867-876. DOI:10.116/compstruc. 2016.10 .120$.

6. L. Kiss and Gy. Szeidl. "Stresses in curved beams made of heterogeneous materials." International Journal of Mechanical Systems of Engineering, 1(2), (2015), p. 8 id. 107. DOI: 10.15344/2455-7412/2015/107.

7. I. Ecsedi and Á. J. Lengyel. "An analytical solution for static problems of curved composit beams." Curved and Layered Structures, 6(1), (2019), pp. 105-116. DOI: 10.1515/cls-2019-0009.

8. L. Ascione and F. Fratenadi. "A penalty model for the analysis of curved composite beams." Computers and Structures, 45(5-6), (1992), pp. 985-999. DOI: 10.1016/0045-7949(92)90057-7.

9. A. Ibrahimbegoric and F. Frey. "Finite element analysis on linear and non-linear planar deformationof elastic initially curved beams." International Journal for Numerical Methods in Engineering, 36(9), (1993), pp. 3239-3528. DOI: 10.1002/ nme.1620361903.

10. G. J. Kim. "An effective composite laminated curved beam element." Communications in Numerical Methods in Engineering, 22(6), (2006), pp. 453-466. DOI: $10.002 / \mathrm{cnm} .829$.

11. I. Ecsedi and K. Dluhi. "A linear model for the static and dynamic analysis of non-homogenous curved beams." Applied Mathematical Modelling 29(12), (2005), pp. 1211-1231. DOI: 10.1016/j.apm.2005.03.006.

12. I. S. Sokolnikoff. Mathematical Theory of Elasticity. McGraw-Hill, 1956. DoI: $10.2307 / 3608899$

13. A. D. Saade. Elastic Theory and Applications. Pergamon Press Inc., 1974. DOI: 10.1016/C2013-0-02524-8.

14. E. Kamke. "Differentialglechungen. Lösungsmethoden and Lösungen I-II." Akademische Verlagsgesellschaft Gest and Portig K.-G. Leipzig, 1951.

15. A. R. Forsyth. A Treatise on Differential Equations. Macmillan, London, 1929. 